\title{
Prevalence of Diplopia and Extraocular Movement Limitation according to the Location of Isolated Pure Blowout Fractures
}

\author{
Min Seok Park, Young Joon Kim, Hoon Kim, Sang Hyun Nam, Young Woong Choi \\ Department of Plastic and Reconstructive Surgery, Sanggye Paik Hospital, Inje University College of Medicine, Seoul, Korea
}

Background Isolated pure blowout fractures are clinically important because they are the main cause of serious complications such as diplopia and limitation of extraocular movement. Many reports have described the incidence of blowout fractures associated with diplopia and limitation of extraocular movement; however, no studies have statistically analyzed this relationship. The purpose of this study was to demonstrate the correlation between the location of isolated pure blowout fractures and orbital symptoms such as diplopia and limitation of extraocular movement.

Methods We enrolled a total of 354 patients who had been diagnosed with isolated pure blowout fractures, based on computed tomography, from June 2008 to November 2011. Medical records were reviewed, and the prevalence of extraocular movement limitations and diplopia were determined.

Results There were 14 patients with extraocular movement limitation and 58 patients complained of diplopia. Extraocular movement limitation was associated with the following findings, in decreasing order of frequency: floor fracture (7.1\%), extended fracture (3.6\%), and medial wall (1.7\%). However, there was no significant difference among the types of fractures $(P=0.60)$. Diplopia was more commonly associated with floor fractures $(21.4 \%)$ and extended type fractures (23.6\%) than medial wall fractures (10.4\%). The difference was statistically significant (Bonferroni-corrected chi-squared test $\mathrm{P}<0.016$ ).

Conclusions Data indicate that extended type fractures and orbital floor fractures tend to cause diplopia more commonly than medial wall fractures. However, extraocular movement limitation was not found to be dependent on the location of the orbital wall fracture.

Keywords Orbital fractures / Eye movements / Diplopia / Chi-squared distribution
Correspondence: Young Woong Choi Department of Plastic and Reconstructive Surgery, Sanggye Paik Hospital, Inje University College of Medicine, 1342 Dongil-ro, Nowon-gu, Seoul 139-707, Korea

Tel: $+82-2-950-1048$

Fax: +82-2-932-6373

E-mail: pshero2@naver.com

This work was supported by a grant from Inje University in 2005.

This article was presented at the 68th Congress of the Korean Society of Plastic and Reconstructive Surgeons on November 4-7, 2010 in Seoul, Korea.

No potential conflict of interest relevant to this article was reported.

Received: 31 Jan $2012 \bullet$ Revised: 9 Mar 2012 • Accepted: 9 Mar 2012

pISSN: 2234-6163 • elSSN: 2234-6171 • http://dx.doi.org/10.5999/aps.2012.39.3.204• Arch Plast Surg 2012;39:204-208

\section{INTRODUCTION}

Isolated pure blowout fractures are caused by an abrupt external impact that leads to a fracture of part of the orbital wall without involving the orbital rim [1]. Although they often present as a simple orbital fracture, immediate diagnosis and treatment are important because acute symptoms, which include diplopia, extraocular movement limitation, and chronic symptoms such as enophthalmos and ectropion, can persist even after surgical treatment $[2,3]$. 
Han et al. [4] reported on blowout fractures in their clinical study, which was conducted over the course of a year and involved only 54 cases of pure and impure blowout fractures. Lee et al. [5] proposed a classification method for blowout fractures using computed tomography findings as well as preoperative and postoperative symptoms including diplopia, extraocular movement limitation, and enophthalmos. However, that study only involved 72 pure blowout fractures over a five-year period. Burm et al. [1] reported on data collected over a four-year period regarding pure blowout fractures and preoperative symptoms. That study only included 83 orbits of 76 patients. Eun et al. [6] presented a large study about blowout fractures, which included 387 patients over a fifteen-year interval. That study provided detailed data on several aspects of the fractures; however, isolation of pure blowout fractures was incomplete. These studies indicated an association between diplopia or extraocular movement limitation and blowout fractures; however, four of the studies included both pure blowout fractures and impure blowout fractures in their analysis. In addition, several studies that investigated blowout fractures simply listed the symptoms by percentages without conducting statistical analyses. Judgments based only on the percentages could be misleading to inexperienced surgeons.

To the best of our knowledge, there have been no reports in the literature that have demonstrated a statistical correlation between the location of fractures and orbital symptoms. Therefore, our aim here was to clarify the location of orbital fractures and the relationship between diplopia and extraocular movement.

\section{METHODS}

We included 354 patients who had been diagnosed with isolated pure blowout fractures from June 2008 to November 2011. An isolated pure blowout fracture was defined as a fracture of one or more of the internal orbital walls excluding adjacent fractures of the facial bone and the orbital rim. Patients with nasal bone fractures, mandibular fractures, and zygomatic arch fractures, in addition to the isolated pure orbital wall fracture, were also included in the study because we determined that the external forces causing these fractures were different. However, concomitant zygomaticomaxillary fractures with fractures of the orbital rim were excluded.

Diplopia and limitation of extraocular movement were retrospectively investigated by reviewing the initial medical records. Locations of the orbital wall fractures were confirmed with computed tomography. Limitation of extraocular movement and diplopia were determined by consulting ophthalmologists and plastic surgeons. Presence of enophthalmos was checked ( $\geq 2 \mathrm{~mm}$ ).
The fracture site was verified by computed tomography and classified as a medial wall fracture or floor fracture by a bony structure, which is located between the ethmoid sinus and the maxillary sinus. Fractures involving both the medial wall and the floor were categorized as extended type fractures.

To verify the correlation between the site of the fracture, and diplopia and extraocular movement limitation, we used the SPSS ver. 19 (IBM Co., Armonk, NY, USA). In addition, we also used cross-tabulation and the Pearson chi-squared test to assess for a statistically significant difference among the three different fracture location groups. A P-value of less than 0.05 was considered statistically significant.

The Bonferroni-corrected chi-squared test revealed a significant difference between each of the following group pairs: medial wall fracture and floor fracture; floor fracture and extended type fracture; and extended fracture and medial wall fracture. A P-value of less than 0.016 was considered statistically significant.

\section{RESULTS}

The study included a total of 354 subjects ( 295 males and 59 females). The most common fractures encountered were medial wall fractures, followed by floor fractures and extended type fractures. Twenty-two patients $(0.06 \%)$ presented with enophthalmos. Limitation of extraocular movement was detected in 14 patients (3.9\%) and diplopia was encountered in 58 patients (16.4\%). All 14 patients suffering from limitation of extraocular movement also complained of diplopia.

The group of patients that presented with medial wall fractures had 3 patients (1.7\%) with extraocular movement limitation and 18 (10.4\%) with diplopia. The group with extended type fractures had $2(3.6 \%)$ with extraocular movement limitation and 13 (23.6\%) with diplopia. The group with floor fractures had 9 (7.1\%) with extraocular movement limitation and 27 (21.4\%) with diplopia 27.

Extraocular movement limitation was associated most commonly with floor fractures (7.1\%) followed by extended fractures (3.6\%) and medial wall fractures (1.7\%) (Fig. 1); however, differences were not statistically significant $(\mathrm{P}>0.05)$ (Table 1$)$. Diplopia was associated with medial wall fractures $(10.4 \%)$, floor fractures (21.4\%), and extended type fractures (23.6\%) (Fig. 2); the differences were found to be statistically significant $(\mathrm{P}<0.05)$ (Table 1). In addition, the different fracture groups were compared to each other. The Bonferroni-corrected chi-squared test revealed that diplopia was more common in the extended type fracture group and the floor fracture group than the medial wall fracture group; these results were statistically significant (Bonferroni correction $\mathrm{P}<0.016$ ) (Fig. 2). 
Fig. 1. Comparison of incidence of extraocular movement limitation

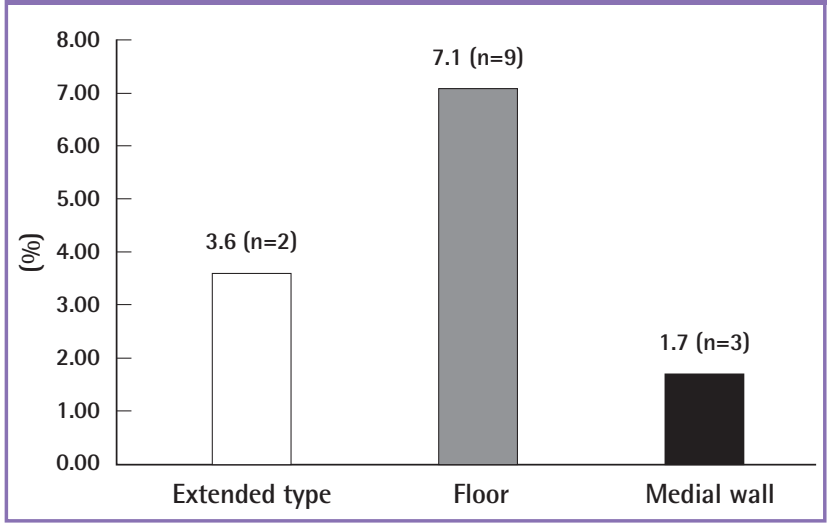

Table 1. Incidence of diplopia and extraocular movement limitation

\begin{tabular}{|lcccc|}
\hline & $\begin{array}{c}\text { Extended } \\
\text { type } \\
(\mathbf{n = 5 5})\end{array}$ & $\begin{array}{c}\text { Floor } \\
(\mathbf{n}=\mathbf{1 2 6})\end{array}$ & $\begin{array}{c}\text { Medial wall } \\
(\mathbf{n}=\mathbf{1 7 3 )}\end{array}$ & $\begin{array}{c}\text { P- } \\
\text { value }^{\text {a) }}\end{array}$ \\
\hline Diplopia & $13(23.6)$ & $27(21.4)$ & $18(10.4)$ & 0.011 \\
EOM limitation & $2(3.6)$ & $9(7.1)$ & $3(1.7)$ & 0.60 \\
\hline $\begin{array}{l}\text { Values are presented as number (\%). } \\
\text { EOM, Extraocular movement. }\end{array}$ & & & \\
a) Chi-squared test. & & & \\
\hline
\end{tabular}

\section{DISCUSSION}

Isolated pure orbital wall fracture is a fracture type with a relatively low incidence rate and is diagnosed in only $13.3 \%$ of all facial bone fractures. However, orbital wall fractures may lead to acute complications such as diplopia, limitation of extraocular movement, globe injury, lacrimal duct injury, retrobulbar hematoma, ptosis, infraorbital nerve anesthesia, and various chronic complications such as ectropion and enophthalmos that can still remain after surgical treatment. Therefore, immediate diagnosis and treatment are important.

Orbital wall fractures occur via the following two main mechanisms: impact on soft tissue and impact on solid orbital rim structures $[7,8]$. The orbital medial wall and floor are fixed to the boundary by a bony buttress, which is located between the ethmoid sinus and the maxillary sinus. Orbital wall fractures are classified as pure blowout fractures and impure blowout fractures based on the involvement of the orbital rim [9]. Most cases of impure blowout fractures are accompanied by orbital rim fractures and may also involve zygomaticomaxillary fractures. Patients with isolated pure blowout fractures were selected for this study, but patients with complex fractures such as zygomaticomaxillary fractures were excluded. However, we included patients with nasal bone fractures without involvement of the
Fig. 2. Comparison of the incidence of diplopia

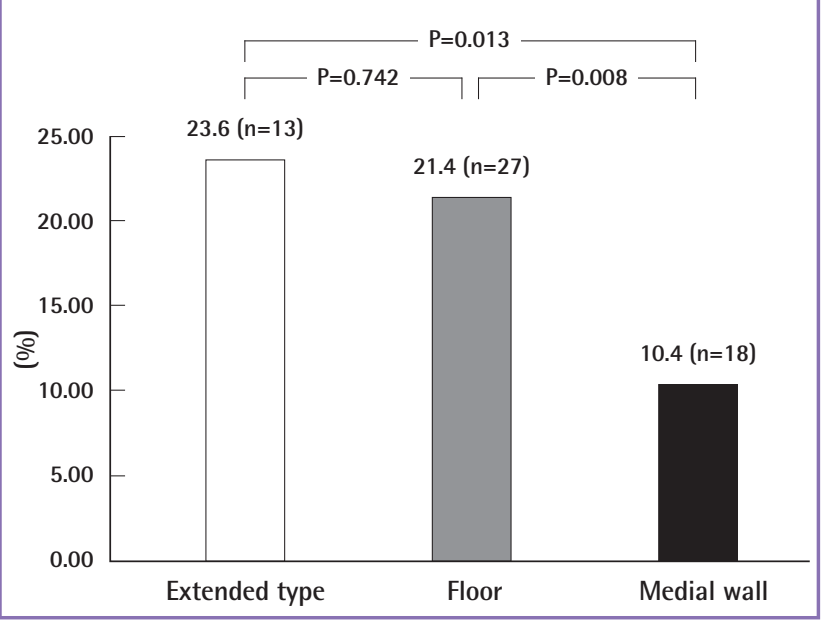

orbital rim and patients with mandibular and zygomatic arch fractures because we determined that the external forces that caused these fractures were different.

A study by Burm et al. [1] included 82 cases and reported that diplopia was associated with $25 \%$ of the medial wall fractures, $80 \%$ of the orbital floor fractures, and $80.9 \%$ of the combined medial and floor fractures. Higashino et al. [10] reported on 106 cases, and showed that $21.4 \%$ of the medial wall fractures and $23.5 \%$ of the orbital floor fractures were associated with diplopia. Eun et al. [6] reported on 387 cases in which diplopia was found on physical examination prior to surgery in $22 \%$ of the medial wall fractures, $78 \%$ of the floor fractures, and $82 \%$ of the combined medial and floor fractures. Tahiri et al. [11] reported that patients with preoperative diplopia had a 9.91 times greater risk of persistent diplopia postoperatively.

Regarding extraocular movement, Burm et al. [1] reported that the incidence of extraocular movement limitation was $12.5 \%$ in medial wall fractures, $73.3 \%$ in floor fractures, and $47.6 \%$ in extended type fractures. Eun et al. [6] reported that the incidence of extraocular movement limitation was $14 \%$ in medial wall fractures, $61 \%$ in floor fractures, and 54\% in extended type fractures.

In contrast, our study revealed low incidence rates for diplopia and extraocular movement limitation, likely because we collected data on only isolated pure blowout fractures and excluded impure blowout fractures. We identified patients with symptoms of diplopia and extraocular movement limitation after both ophthalmologists and plastic surgeons agreed on these findings on physical examination.

The most commonly cited cause for extraocular movement limitation and diplopia is the incarceration of extraocular muscles within the orbital fracture. However, Putterman [12] argued that traumatic hemorrhage leading to swelling of the orbital posterior and inferior orbital fat is the cause. The posterior inferior 
orbital fat, containing a network of venules and bands of fibrous tissue, connects the inferior rectus and inferior oblique muscles to the periosteum of the orbital floor. Swelling of the posterior inferior orbital fat creates strain on the fibrous connective tissue that connects the inferior rectus and the inferior oblique muscles to the orbital floor. Cranial nerve palsies are less frequently the cause of extraocular movement limitation and diplopia. Manson et al. [13] argued that contusions of the inferior muscular branch of cranial nerve III, which supplies the inferior oblique muscle, may be the cause of impaired extraocular movement.

In summary, limitation of extraocular movement and diplopia may be due to intraorbital hemorrhagic swelling, entrapment of the extraocular muscles, tissues, or fat within the fractured bone, or direct damage to the extraocular muscles, nerves, or vasculature [14].

In this study, 5 out of 14 patients that complained of extraocular movement limitation suffered from incarceration of the extraocular muscles. However, Han et al. [4] reported that they could not find incarceration of the inferior rectus muscle or the inferior oblique muscle following blowout fractures in 54 cases.

Converse et al. [2] and Whyte [14] encouraged early surgical treatment to reduce the risk for complications.

The surgical indications of a blowout fracture are as follows: presence of incarceration, radiologic evidence of extensive fracture, significant change of globe position, and visual acuity deficit that needs optic canal decompression [15]. This study used suspicion of incarceration and extensive fracture as viewed on a facial computed tomography as indications for surgery.

In addition to the above mentioned indications, Hwang et al. [16] reported that an intraorbital volume increase of $1 \mathrm{~cm}^{3}$ caused $0.97 \mathrm{~mm}$ of enophthalmos and proposed guidelines for orbital wall reconstruction in blowout fractures which are expected to cause enophthalmos. We included the $2 \mathrm{~cm}^{3}$ increase in the orbital volume as an indication for orbital wall reconstruction in our study. Surgery was performed within 2 weeks after the trauma had occurred and after the swelling had started to disappear [17]. None of the included patients suffered from enophthalmos or visual acuity deficits that required optic canal decompression.

Depending on the location of the fractures, we observed differences in incidence of diplopia but not extraocular movement limitation. Extraocular movement limitation was more commonly associated with orbital floor fractures than with orbital medial wall blowout fractures and extended type fractures. However, these differences were not statistically significant. In contrast, diplopia was associated more frequently with extended type fractures than with medial wall fractures and orbital floor fractures. These differences were statistically significant.

Although there are many reports that show diplopia and extra- ocular movement limitation are often associated with blowout fractures, we did not find a study that analyzed the relationship between the location of pure isolated blowout fractures and the associated symptoms. In this study, we present the correlation between the location of pure isolated blowout fractures and the associated symptoms, diplopia and extraocular movement limitation.

\section{REFERENCES}

1. Burm JS, Chung $\mathrm{CH}, \mathrm{Oh}$ SJ. Pure orbital blowout fracture: new concepts and importance of medial orbital blowout fracture. Plast Reconstr Surg 1999;103:1839-49.

2. Converse JM, Smith B, Obear MF, et al. Orbital blowout fractures: a ten-year survey. Plast Reconstr Surg 1967;39:20-36.

3. Biesman BS, Hornblass A, Lisman R, et al. Diplopia after surgical repair of orbital floor fractures. Ophthal Plast Reconstr Surg 1996;12:9-16.

4. Han JS, Koo SH, Han SK, et al. Blow-out Fracture: clinical study of surgically treated 54 cases. J Korean Soc Plast Reconstr Surg 1995;22:1138-46.

5. Lee JH, Ryu MH, Kim YH. Classification of blowout fracture. J Korean Soc Plast Reconstr Surg 2007;34:719-23.

6. Eun SC, Heo CY, Baek RM, et al. Survey and review of blowout fractures. J Korean Soc Plast Reconstr Surg 2007;34: 599-604.

7. Waterhouse $\mathrm{N}$, Lyne J, Urdang $\mathrm{M}$, et al. An investigation into the mechanism of orbital blowout fractures. Br J Plast Surg 1999;52:607-12.

8. Ahmad F, Kirkpatrick NA, Lyne J, et al. Buckling and hydraulic mechanisms in orbital blowout fractures: fact or fiction? J Craniofac Surg 2006; 17:438-41.

9. Cramer LM, Tooze FM, Lerman S. Blowout fractures of the orbit. Br J Plast Surg 1965;18:171-9.

10. Higashino T, Hirabayashi S, Eguchi T, et al. Straightforward factors for predicting the prognosis of blow-out fractures. J Craniofac Surg 2011;22:1210-4.

11. Tahiri Y, Lee J, Tahiri M, et al. Preoperative diplopia: the most important prognostic factor for diplopia after surgical repair of pure orbital blowout fracture. J Craniofac Surg 2010;21:1038-41.

12. Putterman AM. Management of orbital floor blowout fractures. Adv Ophthalmic Plast Reconstr Surg 1987;6:281-5.

13. Manson PN, Clifford CM, Su CT, et al. Mechanisms of global support and posttraumatic enophthalmos: I. The anatomy of the ligament sling and its relation to intramuscular cone orbital fat. Plast Reconstr Surg 1986;77:193-202.

14. Whyte DK. Blowout fractures of the orbit. Br J Ophthalmol 
1968;52:721-8.

15. Mathes SJ, Hentz VR. Plastic surgery. 2nd ed. Philadelphia: Saunders Elsevier; 2006.

16. Hwang WB, Bae YC, Jeon JY, et al. Orbital volume change in post-traumatic enophthalmos. J Korean Soc Plast Reconstr
Surg 1997;24:1031-43.

17. Huh SH, Yang WY, Hong SP, et al. A study on the clinical improvement according to the fracture sites pure blow-out fracture. J Korean Soc Plast Reconstr Surg 1998;25:1060-6. 